\title{
Paediatric periorbital cellulitis and its management: A ten-year review
}

\author{
MG Alfiky, S Meghji, A Bath \\ Department of Otolaryngology-Head \& Neck Surgery, norfolk and norwich university \\ hospital
}

\section{Background}

Periorbital cellulitis is a relatively common condition in the paediatric population which can lead to devastating morbidity or mortality. Appropriate management and early diagnosis are critical to avoid vision loss, optic neuropathy, cavernous sinus thrombosis, brain abscess, and death.

Chandler et al.4 first described the classification of orbital infections in 1970 that is still referenced today.

\section{Objectives}

1. To analyse the epidemiological, clinical and therapeutic aspects of periorbital cellulitis/abscess in children.

2. To Audit the effectiveness of a locally developed guideline for the management of periorbital cellulitis in children (fig. 1)

\section{Methods}

A retrospective case notes review of paediatric patients who presented to a tertiary centre with any stage of periorbital cellulitis between 2006 -2016.

247 patients were initially identified, then 72 were excluded due to miscoding.

\section{Results}

175 patients were analysed. Age ranged from 1 month to 16 years old. The mean age is 6 years old with female to male ratio of $1: 1.09$. there was only one case presented with bilateral eye involvement. The average annual number of cases was 15.9. the incidence of periorbital infections declined with age. The majority of patients (143) were admitted and only 32 cases were treated as outpatient. Nine patients received outpatient IV antibiotics. The average hospital stay was 3.3 days, with the maximum of 10 days.

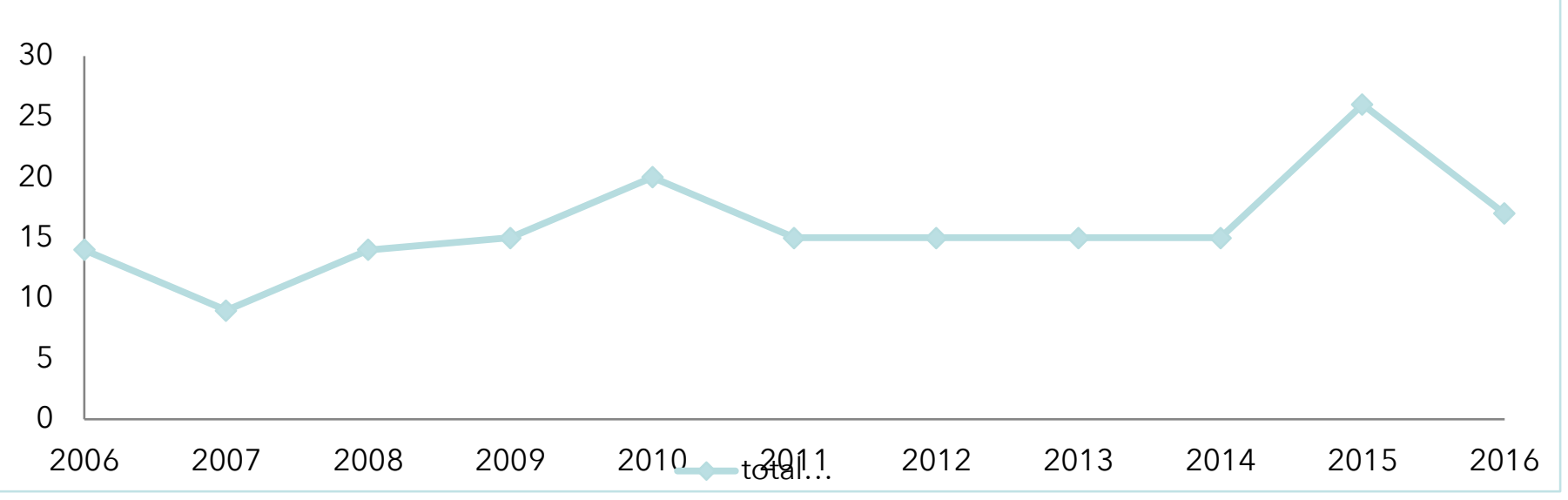

\section{Annual incidence}

ENT and Paeds Ophthal and $2 \%$ ENT

$1 \%$

Paeds and Ophthal $34 \%$

$\begin{array}{ccc}\text { Paediatrici } & \text { ENT } & \text { Ophthal } \\ \text { ans alone } & \text { alone } & \text { alone } \\ 4 \% & 1 \% & 1 \%\end{array}$

Blood culture showed no growth in $93.5 \%$ of cases, while $\mathrm{H}$. Influenza, Staph and Strept are the commonly isolated organisms in positive cultures.

Imaging was done in 53 patients only and 31 of these cases underwent surgical drainage.

Endoscopic approach was used in 16 cases, while 3 cases only were drained externally. Combination of both approaches was done in 9 cases only. Interestingly, 3 cases underwent Endoscopic sinus drainage of infected sinuses due to lack of improvement after IV antibiotic therapy.

\section{Conclusion}

Early and aggressive, MDT management of periorbital infections can prevent the serious possible complications.
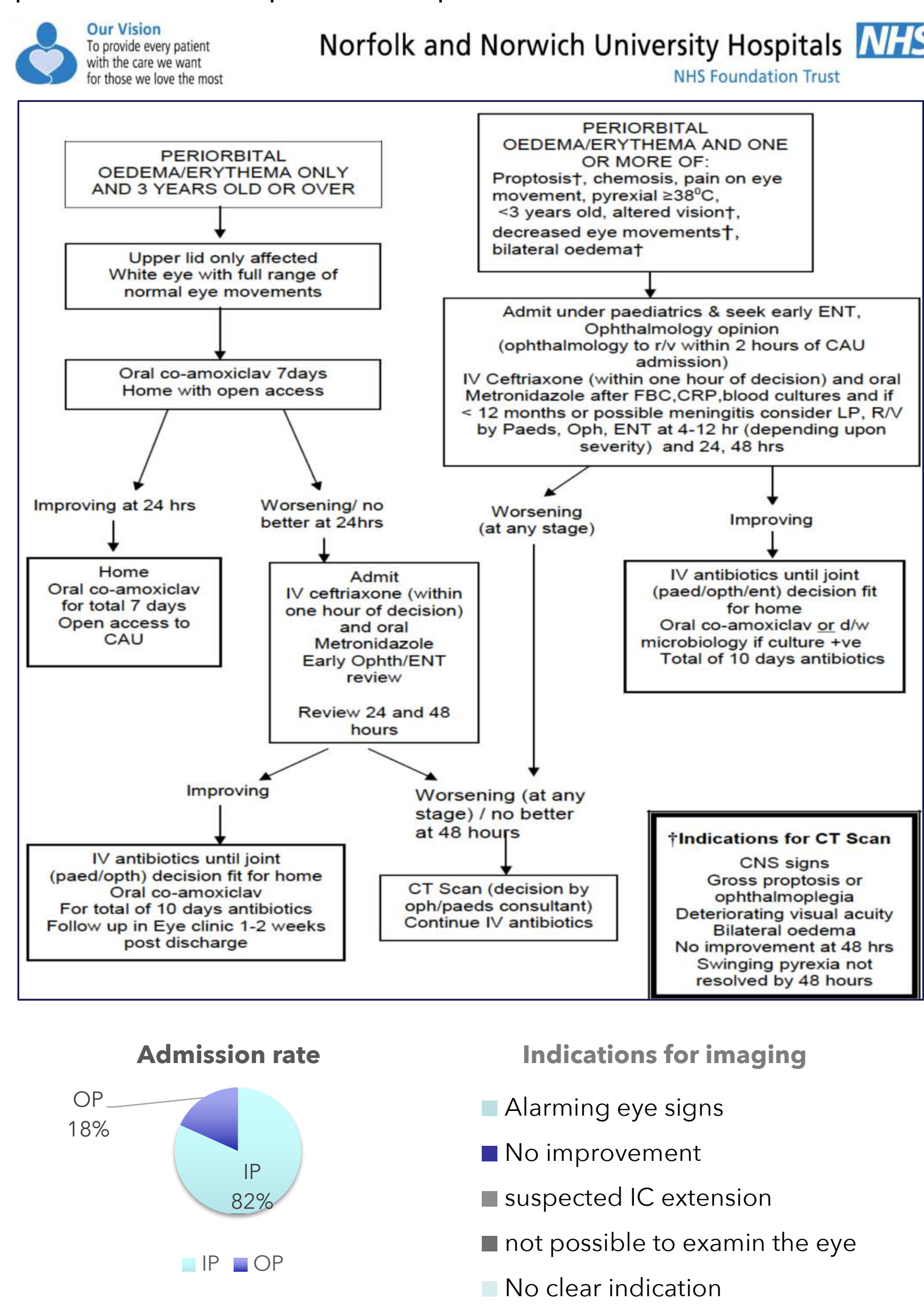